\title{
Endothelial Leukocyte Adhesion Molecule-1 Mediates Antigen-induced Acute Airway Inflammation and Late-phase Airway Obstruction in Monkeys
}

\author{
Robert H. Gundel," Craig D. Wegner," Carol A. Torcellini, ${ }^{\star}$ Cosmos C. Clarke, \\ Nancy Haynes, ${ }^{\ddagger}$ Robert Rothlein, ${ }^{\ddagger}$ C. Wayne Smith," and L. Gordon Letts* \\ Departments of ${ }^{*}$ Pharmacology and ${ }^{\ddagger}$ Immunology, Boehringer Ingelheim Pharmaceuticals, Inc., Ridgefield, Connecticut 06877; \\ and ${ }^{\S}$ Department of Pediatrics, Section of Leukocyte Biology, Baylor College of Medicine, Houston, Texas 77054
}

\begin{abstract}
This study examines the role of endothelial leukocyte adhesion molecule-1 (ELAM-1) in the development of the acute airway inflammation (cell influx) and late-phase airway obstruction in a primate model of extrinsic asthma. In animals sensitive to antigen, a single inhalation exposure induced the rapid expression of ELAM-1 $(6 \mathrm{~h})$ exclusively on vascular endothelium that correlated with the influx of neutrophils into the lungs and the onset of late-phase airway obstruction. In contrast, basal levels of ICAM-1 was constitutively expressed on vascular endothelium and airway epithelium before antigen challenge. After the single antigen exposure, changes in ICAM-1 expression did not correlate with neutrophil influx or the change in airway caliber. This was confirmed by showing that pretreatment with a monoclonal antibody to ICAM-1 did not inhibit the acute influx of neutrophils associated with late-phase airway obstruction, whereas a monoclonal antibody to ELAM-1 blocked both the influx of neutrophils and the late-phase airway obstruction. This study demonstrates a functional role for ELAM-1 in the development of acute airway inflammation in vivo. We conclude that, in primates, the late-phase response is the result of an ELAM-1 dependent influx of neutrophils. Therefore, the regulation of ELAM-1 expression may provide a novel approach to controlling the acute inflammatory response, and thereby, affecting airway function associated with inflammatory disorders, including asthma. (J. Clin. Invest. 1991. 88:14071411.) Key words: cellular adhesion molecules $\bullet$ airway inflammation • airway obstruction $\bullet \operatorname{monoclonal}$ antibody $\bullet \operatorname{monkeys}$
\end{abstract}

\section{Introduction}

It is well documented that inhalation of allergens by susceptible asthmatics causes the occurrence of an immediate airway obstruction that is maximal at 10-20 min and resolves by $1-2 \mathrm{~h}$ post-antigen exposure. Many also experience a second, late asthmatic response characterized by prolonged bronchial ob-

Address correspondence to Robert H. Gundel, Ph.D., Department of Pharmacology, Boehringer Ingelheim Pharmaceuticals, Inc., 90 East Ridge/P.O. Box 368, Ridgefield, CT 06877.

Received for publication 08 May 1991 and in revised form 27 June 1991.

J. Clin. Invest.

(C) The American Society for Clinical Investigation, Inc.

$0021-9738 / 91 / 10 / 1407 / 05 \$ 2.00$

Volume 88, October 1991, 1407-1411 struction, the onset of which usually begins 4-5 $\mathrm{h}$ after initial allergen exposure and may persist for up to $24 \mathrm{~h}$ or more $(1,2)$.

The development of airway inflammation and the role of proinflammatory cells in the clinical manifestations of asthma have become the subject of profound investigation. In particular, many recent studies have suggested an active role for nonresident proinflammatory cells that actively infiltrate the lung and selectively release on specific target tissue, potent preformed and/or newly generated proinflammatory mediators that contribute to the late-phase response $(3,4)$.

The accumulation of polymorphonuclear leukocytes at sites of inflammation is dependent upon a complex series of events. Of particular interest is the upregulation and enhanced expression of cell surface adhesion glycoproteins on vascular endothelium and circulating leukocytes leading to leukocyte adherence to the vessel wall, activation, and subsequent extravasation. In the present study we have examined the function of two vascular adhesion molecules, intercellular adhesion molecule-1 (ICAM-1) 1 and endothelial leukocyte adhesion molecule-1 (ELAM-1), in the development of antigen-induced acute airway inflammation and the associated late-phase airway obstruction in primates.

\section{Methods}

Animals. The animals used in this study were wild-caught adult male cynomolgus monkeys (Macaca fascicularis) weighing $\sim 4-8 \mathrm{~kg}$ (Charles River Breeding Laboratories, Inc., Primate Imports, Port Washington, NY). Each animal was housed individually in a specially designed open mesh cage and provided with food twice a day and water ad libitum.

Study protocol. Each animal was anesthetized with an intramuscular injection of ketamine ( $4 \mathrm{mg} / \mathrm{kg}$; Ketaset; Myoderm Medical Supply, Norristown, PA) and xylazine ( $1 \mathrm{mg} / \mathrm{kg}$; Rompun; Miles Laboratories, Inc., Naperville, IL), intubated with a cuffed endotracheal tube, and placed in the supine position. Ketamine $(4 \mathrm{mg} / \mathrm{kg}$, i.m.) was used as supplemental anesthesia as needed. Each animal then received a bolus intravenous injection of monoclonal antibody or vehicle (saline). Airway and tissue cellular composition was then evaluated by performing bronchoalveolar lavage (BAL) with a pediatric fiberoptic bronchoscope and bronchial biopsy, after which the animals were seated in the upright position in a specially designed support chair. Baseline respiratory system resistance (Rrs) was monitored for $\sim 15$ min followed by an inhaled antigen challenge ( $1 \mathrm{~h}$ post-iv treatment). Rrs was monitored continuously for $1 \mathrm{~h}$ after which the animals were allowed to recover from anesthesia and returned to their cages. At 4, 6, 8, and $10 \mathrm{~h}$

1. Abbreviations used in this paper: BAL, bronchoalveolar lavage; ELAM-1, endothelial leukocyte adhesion molecule-1; ICAM-1, intercellular adhesion molecule-1; Rrs, respiratory system resistance. 
after antigen inhalation the animals were anesthetized (ketamine/xylazine), intubated, and Rrs was monitored over a 15-min time period to record the late-phase response. Cellular influx into the lungs during the peak late-phase response was assessed by performing BAL (opposite lung lavaged before antigen challenge) and bronchial biopsy.

The study was designed such that bracketing control experiments (vehicle treatment) were performed on each animal such that each animal represents its own control. Each study was separated by $14 \mathrm{~d}$.

$M A b s$. An anti-human ELAM-1 MAb was prepared by immunizing $B A L B / c$ mice with human umbilical vein endothelial cells (HUVEC) isolated and cultured as previously described (5), and stimulated with IL-1 $(3 \mathrm{U} / \mathrm{ml})$ for $4 \mathrm{~h}$. The immunization schedule and preparation of hybridomas was performed as previously described (6), and clone selection was based on the detection of newly expressed endothelial antigens after $4 \mathrm{~h}$ stimulation of HUVEC with $3 \mathrm{U} / \mathrm{ml} \mathrm{IL}-1$. One MAb selected for the present study, CL2, was found to recognize selectively $C O S$ cells and $L$ cells transfected with ELAM- 1 cDNA but not LFA-3 or ICAM-1 cDNA (Kishimoto, T. K., R. A. Warnock, M. A. Jutila, E. C. Butcher, C. L. Lane, D. C. Anderson, and C. W. Smith, submitted for publication). This MAb also immunoprecipitated a 115kD surface glycoprotein from IL-1-stimulated HUVEC, and in adhesion studies in vitro, significantly inhibited the adhesion of CD18-deficient human neutrophils to IL-1-stimulated HUVEC. The binding pattern of CL2 was tested with monkey leukocytes and found to weakly bind to neutrophils and lymphocytes. However, this antibody does not inhibit neutrophil or lymphocyte function (i.e., aggregation, chemotaxis). In addition, intravenous treatment with the MAbs does not alter the circulating white cell count as previously described (7).

Antibodies against ICAM-1 were generated as previously described (7). Stock MAb solutions were diluted with saline (concentration, 2 $\mathrm{mg} / \mathrm{ml}$ ) immediately before intravenous injection into a peripheral leg vein. MAb treatment was administered $1 \mathrm{~h}$ before antigen inhalation challenge. This timing was based on previous work (7) where we showed functional activity with these monoclonol antibodies.

Rrs measurements. Respiratory system impedance $(\mathrm{Zrs})$ was measured by discrete frequency (4-40 $\mathrm{Hz}$ in 11 equal logarithmic steps) sinusoidal forced oscillations superimposed on tidal breathing as previously described (8). The mean of the real or in-phase component of $\mathrm{Zrs}$ over the entire frequency range was then computed to provide a single value representation of Rrs.

Bronchoalveolar lavage $(B A L)$. BAL was performed by guiding a fiberoptic bronchoseope (model 3C-10; Olympus Optical, Lake Success, NY) past the carina and wedged into a fifth- to seventh-generation bronchus. A 15-ml aliquot of bicarbonate buffered saline (pH 7.4, $23^{\circ} \mathrm{C}$ ) was infused and gently aspirated through a channel in the bronchoscope. Collected samples were centrifuged at $2,000 \mathrm{rpm}$ for $10 \mathrm{~min}$ and the resulting cell pellets were resuspended in $\mathrm{Ca}^{++}$and $\mathrm{Mg}^{++}$free HBSS. We have found previously that the BAL procedure will elicit a mild inflammatory response. Thus, to avoid the possible effects of BAL on lung cellular composition, BAL was performed alternating right and left lungs before and after antigen challenge. The return volume of infused buffer was very constant throughout the study and the procedure was well tolerated by the animals. Total white cell counts were obtained using a Coulter counter (model No. 10; Coulter Electronics, Inc., Hialeah, FL).

Antigen inhalation challenge. Antigen inhalation challenges were administered by intermittent positive pressure breathing with a Mark 7A respirator and micronebulizer (model No. 8158; Bird Corp., Palm Springs, CA). Each challenge consisted of 15 breaths-per-minute (maximum inspiratory pressure of $20 \mathrm{~cm} \mathrm{H}_{2} \mathrm{O}$ ) for $2 \mathrm{~min}$. Ascaris suum extract (Greer Laboratories, Lenoir, NC) was diluted with PBS (pH 7.4) to the appropriate concentration for each animal. The concentration of Ascaris suum extract was titrated for each animal to obtain reproducible early- and late-phase responses. Antigen challenges were separated by $14 \mathrm{~d}$ for each animal. Each animal was fasted for $18 \mathrm{~h}$ before the day of study.

Histochemistry. The typing of BAL cells were evaluated using cytocentrifuge preparations stained with Diff-Quick stain (Fisher Scientific
Co., St. Louis, MO). Differential cell counts were determined by counting 200 cells and the percentages of each cell type was recorded.

Histology. Lung biopsy samples were obtained before antigen challenge and during the peak late-phase response with biopsy forceps and the fiberoptic bronchoscope. Immunohistochemical staining for identification of ICAM-1 and ELAM-1 on pulmonary vascular endothelium and airway epithelium was performed as previously described (7).

Statistical analysis. Data were analyzed statistically using a twoway analysis of variance and Friedman's multiple range test.

\section{Results}

In the first series of experiments we examined the lung cellular composition, by BAL, before and at several time points after a single antigen challenge (Fig. 1). Before antigen challenge there were a large number of eosinophils recovered by BAL. At $6 \mathrm{~h}$ postchallenge (during the peak late-phase airway obstruction) the number of eosinophils decreased but returned to prechallenge values by day 1 . Eosinophils remained chronically elevated at days 7 and 14. Neutrophils, however, comprised a very small percentage of BAL cells before antigen challenge and were dramatically increased at the peak of the late-phase airway obstruction $(6 \mathrm{~h})$. Neutrophils returned to basal levels by day 1 and remained low at days 7 and 14. The influx of neutrophils correlated significantly with the magnitude of the latephase response $(r=0.61 ; P<0.05)$.

Immunohistochemical staining of lung tissue revealed positive staining for ICAM-1 on both pulmonary vascular endothelium and airway epithelium $6 \mathrm{~h}$ after inhalation challenge (Fig. 2). In contrast, staining for ELAM-1 was clearly increased only on pulmonary vascular endothelium $6 \mathrm{~h}$ postchallenge. No staining for ELAM-1 was found on airway epithelium.

Pretreatment with the anti-ELAM-1 monoclonal antibody (CL2) $1 \mathrm{~h}$ before antigen inhalation significantly attenuated both the total leukocyte infiltration and the number of infiltrating neutrophils in all animals, markedly in three animals (Fig. 3). Pretreatment with the anti-ICAM-1 monoclonal antibody (R6.5) did not significantly alter the number of infiltrating leu-

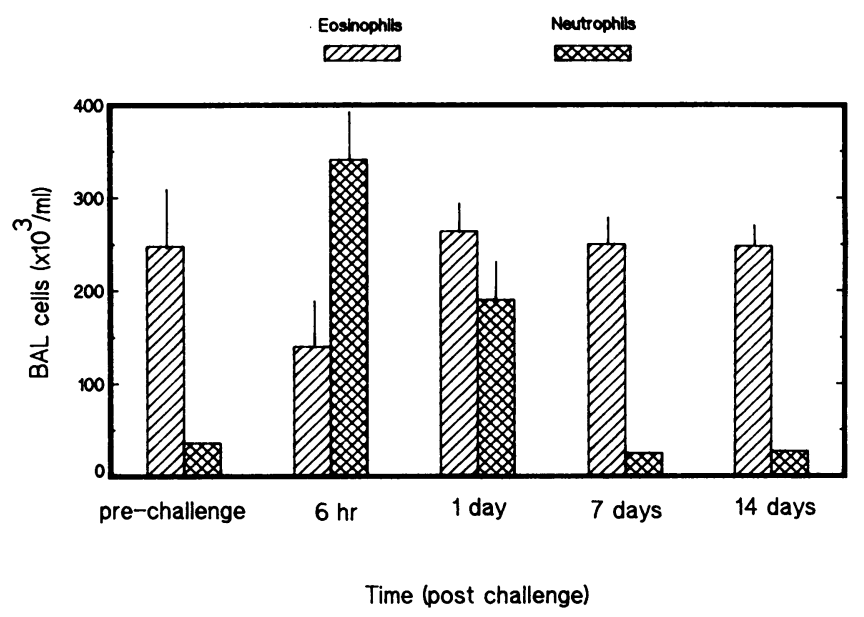

Figure 1. Airway cellular composition before and after antigen inhalation challenge. Note the large number of eosinophils recovered prior to antigen challenge. After challenge the number of eosinophils decreased but returned to prechallenge levels by day 1 and remained elevated at days 7 and 14. Most striking was the large influx of neutrophils associated with the late-phase airway obstruction response, peaking at $6 \mathrm{hr}$ and returning to prechallenge values by day 7 . 

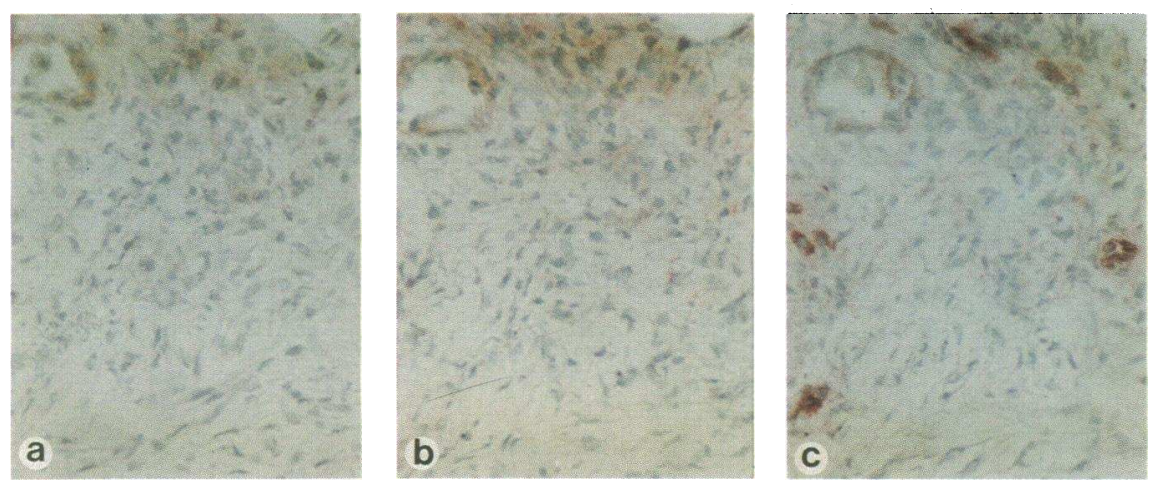

Figure 2. Immunohistochemical staining with (A) normal mouse serum, (B) antibody to ICAM-1 and (C) antibody to ELAM-1 on lung tissue obtained by biopsy $6 \mathrm{hrs}$. post challenge. ICAM-1 staining was found on both airway epithelium and vascular endothelium. In contrast, staining for ELAM-1 was evident only on airway vascular endothelium.

kocytes recovered by BAL or the neutrophil influx (Fig. 4). CL2 treatment resulted in a significant reduction in the latephase response, whereas R6.5 had no effect (Fig. 5). Neither treatment had an effect on the early response.

\section{Discussion}

Adhesion molecules are critical in the extravasation of neutrophils to sites of inflammation. The need for the CD18 integrins is clearly shown by the fact that neutrophil infiltration in infected lesions is almost absent in patients with CD18 deficiency (9). The use of anti-CD18 and anti-ICAM-1 MAbs in various animal models has also demonstrated that CD18 integrins and ICAM-1 are needed for neutrophil emigration at many inflammatory sites (10-12). In addition though, recent evidence indicates that CD18-independent mechanisms are required for emigration of neutrophils in some inflammatory conditions in the lungs of rabbits and humans $(10,13)$. Studies in vitro show that CD18-independent neutrophil-endothelial adhesion involves members of the LEC-CAM family of adhesion molecules (1419). Though numerous investigations indicate a role for ELAM-1 in adhesive interactions of both lymphocytes and neu- trophils and cytokine-stimulated endothelial cells in vitro (15, 20-24), its role in vivo has not been directly assessed. ELAM-1 is found on human venular endothelial cells at sites of acute (25) and chronic (24) inflammation, and in primates at cutaneous sites of cytokine injection (26). Though, it is unclear what role ELAM-1 plays in chronic inflammation, its presence at skin inflammatory sites with an infiltrate of predominantly mononuclear leukocytes is consistent with observations in vitro that ELAM-1 supports adherence of some lymphocytes (23, 24). The results in the present report are the first showing that ELAM-1 participates in acute neutrophil extravasation and injury in antigen-induced airway inflammation in vivo.

The rapidly developing airway neutrophilia associated with late-phase airway obstruction occurs largely via an endothelial ELAM-1-dependent process. This observation is supported by immunohistochemical staining of lung tissue obtained by biopsy before and after antigen challenge that demonstrated a marked upregulation and enhanced expression of ELAM-1 exclusively on vascular endothelium. Other studies in vitro have demonstrated that ELAM-1 is not constitutively expressed but is rapidly upregulated and maximally expressed $4-6 \mathrm{~h}$ postcytokine (i.e., IL-1) stimulation (27). These studies have shown
A

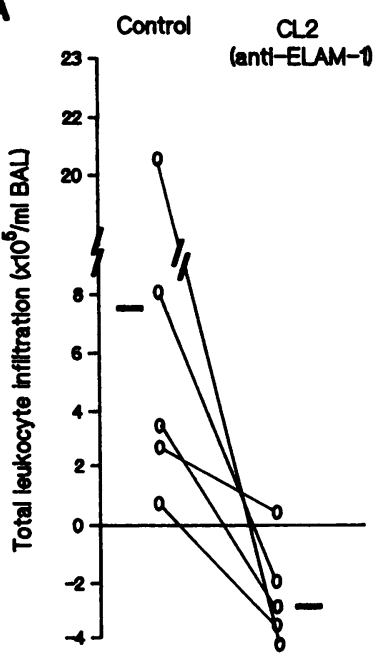

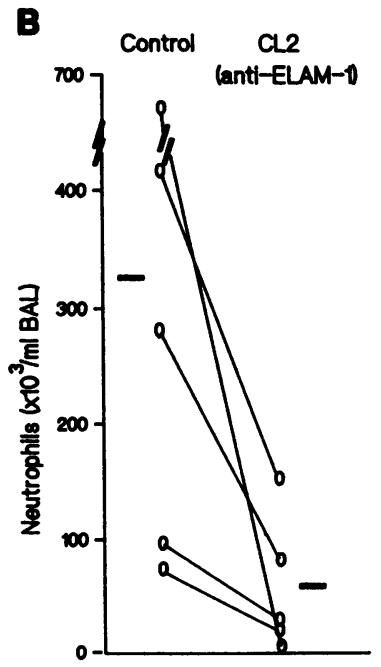

Figure 3. The recovery of total leukocytes (A) and neutrophils (B) during control (vehicle treatment) and anti-ELAM-1 (CL2) treated animals. CL2 (2 mg/kg, i.v.) significantly attenuated the total number of leukocytes as well as the number of neutrophils infiltrating into the lungs $6 \mathrm{hrs}$ after antigen inhalation.
A
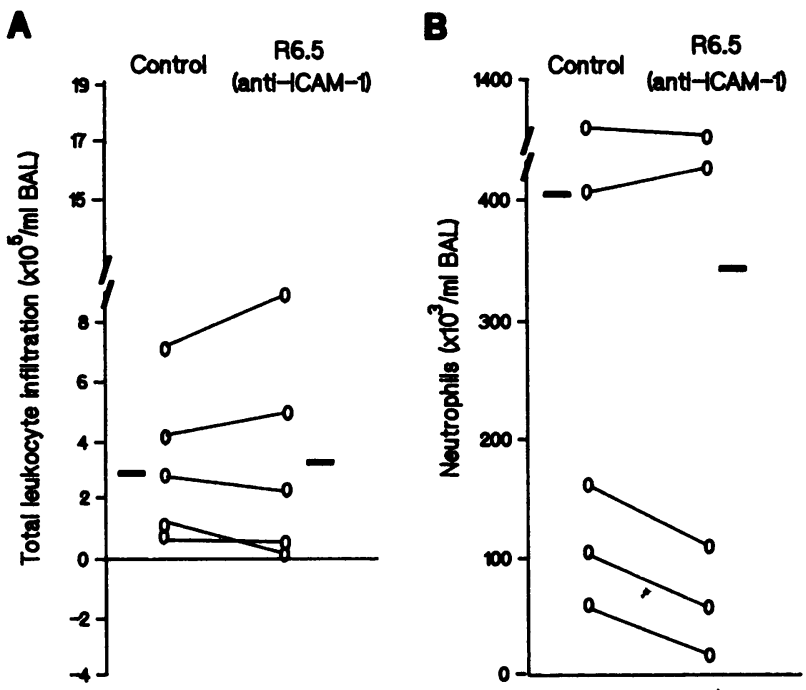

Figure 4. The effects of anti-ICAM-1 (R6.5) treatment $(2 \mathrm{mg} / \mathrm{kg}$, i.v.) on the $(A)$ total number of leukocytes and $(B)$ neutrophils recovered by BAL $6 \mathrm{~h}$ postantigen inhalation. R6.5 did not significantly reduce the total number of leukocytes or neutrophils infiltrating into the airways after antigen inhalation. 


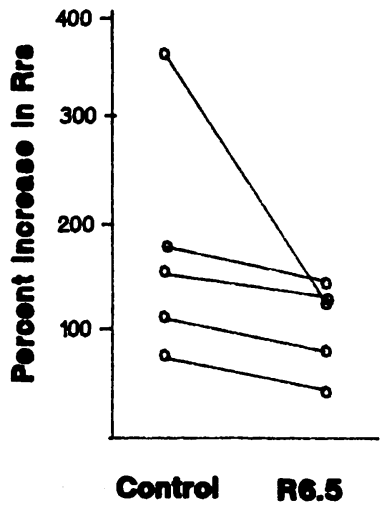

B

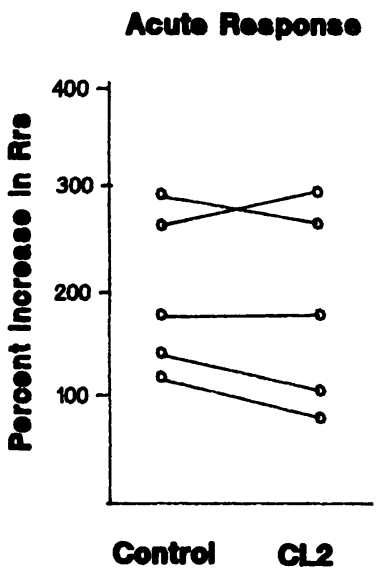

Lato-Phase Roeponse

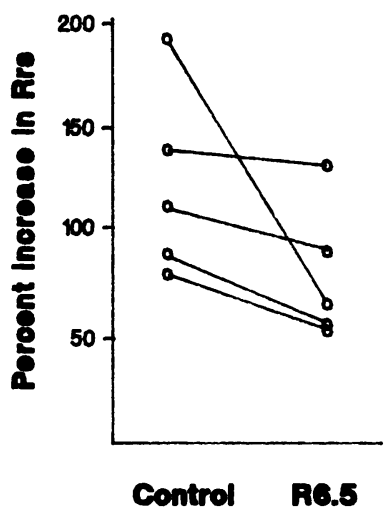

LaterPhase Reeponse

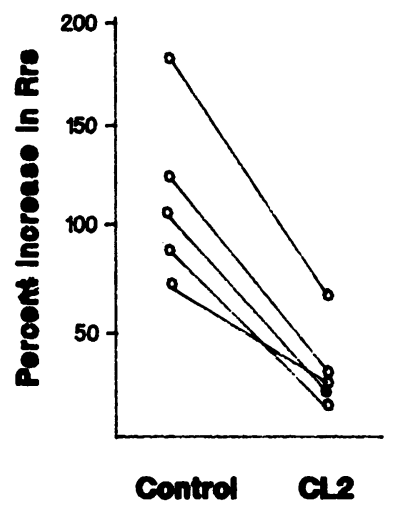

Figure 5. The effects of $(A)$ anti-ICAM-1 (R6.5) or $(B)$ anti-ELAM-1 (CL2) treatment on the immediate and late-phase response. CL2 significantly inhibited the late-phase response, whereas R6.5 had no effect. Neither treatment significantly effected the immediate airway obstruction response. that ELAM-1 expression is transient and returns to basal levels by $16-24 \mathrm{~h}$. In contrast, ICAM-1 is constitutively expressed but is also upregulated by cytokine stimulation with $\sim 50 \%$ maximal expression at 4-6 $\mathrm{h}$ and maximal expression at 16-24 $\mathrm{h}$. Thus, the kinetics of ELAM-1 and ICAM-1 expression in vitro are similar to what we have observed in vivo and are supportive for a role of ELAM-1 in the acute airway inflammation in the primate.

The significant correlation of the magnitude of the neutrophil influx with the magnitude of the late-phase bronchoobstruction suggest an effector cell role for the neutrophil in the late-phase response. Further, inhibition of the neutrophil influx at the level of the endothelium and prevention of the latephase obstruction by CL2 provides additional support for a role of the neutrophil in the late-phase response.

Interestingly, treatment with anti-ICAM-1 did not block the neutrophil influx and late-phase response. We conclude from this that ICAM-1 is not the predominant adhesion protein involved in the acute airway inflammatory response. Of note is that we have shown previously that ICAM-1 does play a pivital role in the development of chronic airway inflammation and the associated airway hyperresponsiveness induced with multiple inhalation antigen challenges (7). In these studies R6.5 (anti-ICAM-1) significantly reduced the airway eosinophilia and prevented any increase in airway responsiveness to inhaled methacholine. In contrast to the role of the eosinophil in the chronically inflamed lung, we see little change in the number of neutrophils. In another recent study of chronic airway inflammation (28), we demonstrated that neutrophil influx only occurs during the recovery phase when airway function and hyperresponsiveness are returning to preinflammatory values and, therefore, suggest that the neutrophil has a predominantly reparative role in chronic airway inflammation. Thus, together the data presented suggests a functional role for both ELAM-1 and ICAM-1 in antigen-induced acute and chronic airway inflammation, respectively. Furthermore, data from this study and from previous studies from our laboratory demonstrate important differences in the role of cellular adhesion molecules and proinflammatory cell types in acute or chronic airway inflammation.

\section{References}

1. O'Byrne, P. M., J. Dolovich, and F. E. Hargreave. 1987. Late asthmatic responses. Am. Rev. Respir. Dis. 136:740-745.

2. Larsen, G. L. 1987. The pulmonary late-phase response. Hosp. Pract. 22:155-169.

3. Marsh, W. R., C. G. Irvin, K. R. Murphy, B. L. Behrens, and G. L. Larsen. 1985. Increases in airway reactivity to histamine and inflammatory cells in bronchoalveolar lavage after the late asthmatic response in an animal model. Am. Rev. Respir. Dis. 131:875-879.

4. de Monchy, J. G. R., H. R. Kaufman, P. Venge, G. H. Koeter, H. M. Jansen, H. J. Sluiter, and K. de Vries. 1985. Bronchoalveolar eosinophilia during allergen-induced late asthmatic reactions. Am. Rev. Respir. Dis. 131:373-376. 
5. Smith, C. W., R. Rothlein, B. J. Hughes, M. M. Mariscalco, F. C. Schmalstieg, and D. C. Anderson. 1988. Recognition of an endothelial determinant for CD18-dependent human neutrophil adherence and transendothelial migration. J. Clin. Invest. 82:1746-1756.

6. Smith, C. W., S. D. Marlin, R. Rothlein, C. Toman, and D. C. Anderson. 1989. Cooperative interactions of LFA-1 and Mac-1 with intercellular adhesion molecule- 1 in facilitating adherence and transendothelial migration of human neutrophils in vitro. J. Clin. Invest. 83:2008-2017.

7. Wegner, C. D., R. H. Gundel, P. Reilly, N. Haynes, L. G. Letts, and R. Rothlein. 1990. Intercellular adhesion molecule-1 (ICAM-1) in the pathogenesis of asthma. Science (Wash. DC). 247:456-459.

8. Wegner, C. D., A. C. Jackson, J. D. Berry, and J. R. Gillespie. 1984. Respiratory mechanics in monkeys measured by forced oscillations. Annu. Rev. Physiol. 55:47-61.

9. Anderson, D. C., and T. A. Springer. 1987. Leukocyte adhesion deficiency: an inherited defect in the Mac 1, LFA 1 and p150,05 glycoproteins. Annu. Rev. Med. 38:175-194.

10. Carlos, T. M., and J. M. Harlan. 1990. Membrane proteins involved in phagocyte adherence to endothelium. Immunol. Rev. 114:5-28.

11. Barton, R. W., R. Rothlein, J. Ksiazek, and C. Kennedy. 1989. The effect of anti-intercellular adhesion molecule-1 on phorbol-ester-induced rabbit lung inflammation. J. Immunol. 143:1278-1282.

12. Seewaldt-Becker, E., R. Rothlein, and J. W. Dammgen. 1989. CD18-dependent adhesion of leukocytes to endothelium and its relevance for cardiac reperfusion. In Leukocyte Adhesion Molecules: Structure, Function, and Regulation. T. A. Springer, D. C. Anderson, A. S. Rosenthal, and R. Rothlein, editors. Springer-Verlag, Inc., New York. 138-148.

13. Doerschuk, C. M., R. K. Winn, H. O. Coxson, and J. M. Harlan. 1990. CD18-dependent and independent mechanisms of neutrophil emigration in the pulmonary and systemic microcirculation of rabbits. J. Immunol. 144:23272333.

14. Stoolman, L. M. 1989. Adhesion molecules controlling lymphocyte migration. Cell. 56:907-910.

15. Bevilacqua, M. P., S. Stengelin, Jr., M. A. Gimbrone, and B. Seed. 1989 Endothelial leukocyte adhesion molecule 1: an inducible receptor for neutrophils related to complement regulatory proteins and lectins. Science (Wash. DC). 243:1160-1165.

16. Geng, J., M. P. Bevilacqua, K. L. Moore, T. M. McIntyre, S. M. Prescott J. M. Kim, G. A. Bliss, G. A. Zimmerman, and R. P. McEver. 1990. Rapid neutrophil adhesion to activated endothelium mediated by GMP-140. Nature (Lond.). 343:757-760.

17. Hallmann, R., M. A. Jutila, C. W. Smith, D. C. Anderson, T. K. Kishimoto, and E. C. Butcher. 1991. The peripheral lymph node homing receptor,
LECAM-1, is involved in CD-18-independent adhesion of human neutrophils to endothelium. Biochem. Biophys. Res. Commun. 174:236-243.

18. Smith, C. W., T. K. Kisimoto, O. Abbassi, B. J. Hughes, R. Rothlein, L. V. McIntire, E. Butcher, and D. C. Anderson. 1991. Chemotactic factors regulate lectin adhesion molecule 1 (LECAM-1)-dependent neutrophil adhesion to cytokine-stimulated endothelial cells in vitro. J. Clin. Invest. 87:609-618.

19. Luscinskas, F. W., A. F. Brock, M. A. Arnaout, and M. A. Gimbrone, Jr. 1989. Endothelial-leukocyte adhesion molecule-1-dependent and leukocyte (CD11/CD18)-dependent mechanisms contribute to polymorphonuclear leukocyte adhesion to cytokine-activated human vascular endothelium. J. Immunol. 142:2257-2263.

20. Bevilacqua, M. P., J. S. Pober, D. L. Mendrick, R. S. Cotran, and M. A. Gimbrone, Jr. 1987. Indentification of an inducible endothelial-leukocyte adhesion molecule. Proc. Natl. Acad. Sci. USA. 84:9238-9242.

21. Low, J. B., L. M. Stoolman, R. P. Nair, R. D. Larson, T. L. Berhend, and R. M. Marks. 1990. ELAM-1-dependent cell adhesion to vascular endothelium determined by a transfected human fucosyltransferase cDNA. Cell. 63:475-484.

22. Walz, G., A. Aruffo, W. Kolanus, M. P. Bevilacqua, and B. Seed. 1990. Recognition by ELAM-1 of the Sialyl-Le ${ }^{x}$ determinant on myeloid and tumor cells. Science (Wash. DC). 250:1132-1135.

23. Graber, N., T. V. Gopal, D. Wilson, L. D. Beall, T. Polte, and W. Newman. 1990. T cells bind to cytokine-activated endothelial cells via a novel, inducible sialoglycoprotein and endothelial leukocyte adhesion molecule 1 . J. Immunol. 145:819-830.

24. Picker, L. J., T. K. Kishimoto, C. W. Smith, R. A. Warnock, and E. C. Butcher. 1990. ELAM-1 is an adhesion molecule for skin-homing T-cells. Nature (Lond.). In press.

25. Cotran, R. S., Jr., M. A. Gimbrone, M. P. Bevilacqua, D. L. Mendrick, and J. S. Pober. 1986. Induction and detection of a human endothelial activation antigen in vivo. J. Exp. Med. 164:661-666.

26. Munro, J. M., J. S. Pober, and R. S. Cotran. 1989. Tumor necrosis factor and interferon-gamma induce distinct patterns of endothelial activation and associated leukocyte accumulation in skin of papio anubis. Am. J. Pathol. 135:121133.

27. Pober, J. S., M. P. Bevilacqua, D. L. Mendrick, L. A. Lapierre, W. Fiers and M. A. Gimbrone, Jr. 1986. Two distinct monokines, interleukin 1 and tumor necrosis factor, each independently induce biosynthesis and transient expression of the same antigen on the surface of cultured human vascular endothelial cells. $J$. Immunol. 136:1680-1687.

28. Gundel, R. H., C. D. Wegner, C. A. Torcellini, C. C. Clarke, G. J. Gleich, and L. G. Letts. 1990. Relationship between bronchoalvealar lavage (BAL) eosinphil-derived protein and the onset and recovery of airway hyperresponsiveness. $J$. Allergy Clin. Immunol. 85:282. (Abstr.) 